\title{
Phytosociology with Other Characteristic Biologically and Ecologically of Plant in Palestine
}

\author{
Jehad M. H. Ighbareyeh'1, A. Cano-Ortiz ${ }^{\text {, Asma A. A. Suliemieh }}{ }^{2}$, \\ Mohammed M. H. Ighbareyeh ${ }^{3}$, E. Cano ${ }^{*}$ \\ ${ }^{1}$ Department of Animal and Plant Biology and Ecology, Faculty of Experimental Sciences, University of Jaen, \\ Jaen, Spain \\ ${ }^{2}$ Faculty of Sciences, University of Hebron, Hebron, Palestine \\ ${ }^{3}$ Faculty of Arts, Quds Open University, Hebron, Palestine \\ Email:
}

Received 20 July 2014; revised 25 August 2014; accepted 25 September 2014

Copyright (C) 2014 by authors and Scientific Research Publishing Inc.

This work is licensed under the Creative Commons Attribution International License (CC BY). http://creativecommons.org/licenses/by/4.0/

(c) (i) Open Access

\begin{abstract}
Idna, Hebron area, Palestine was subject to phytosociological study through the period from March to May 2013; this area has a characteristic dry, arid, semi-arid and very little of sub-humid and locates between Mediterranean, Negev and Sinai regions. We took 237 samples of different species plants from Idna village; the absence of phytosociological studies on the area led us to run a statistical treatment on the 237 woody plant inventories. Moreover, the inventories were made following Braun-Blanquet 1979; we transformed the Braun-Blanquet species abundance-dominance values into those of Van der Maarel 1979. In the statistical treatment we obtained two large groups in the cluster: group (A), representing forests, copses and high shrublands influenced by climate (climatophilous); and group (B), representing Tamarix copses which are influenced by edaphohygrophilic. Working a great interest for Palestine. Due to the lack of phytosociological studies in this country. For the first time gets to sample and characterize the phytosociological methodology woody plant communities. The objective of this study is to obtain bioclimatic indicators to trigger sustainable agricultural development. We propose eight association plant communities such as ASL1 = association of woody plants (association one)-Pistacio palaestinae-Quercetum lokii; ASL2-Capparido sinaicae-Ceratonietum siliquae; ASL3-Cerasus microcarpae-Quercetum ithaburensis; ASL4-Pyro siriacae-Abietetum cilicicae; ASL5-Abio ciliciae-Ceratonietum siliquae; ASL6Periploco aphylli-Pinetum halepensis; ASL7-Cytisopsis pseudocytiso-Tamaricetum tetragynae; ASL8Crataego sinaicae-Tamaricetum jordanii.
\end{abstract}

\footnotetext{
${ }^{*}$ Corresponding author.
}

How to cite this paper: Ighbareyeh, J.M.H., Cano-Ortiz, A., Suliemieh, A.A.A., Ighbareyeh, M.M.H. and Cano, E. (2014) Phytosociology with Other Characteristic Biologically and Ecologically of Plant in Palestine. American Journal of Plant Sciences, 5, 3104-3118. http://dx.doi.org/10.4236/ajps.2014.520327 


\section{Keywords}

Palestine, Ecology, Biology, Phytosociology, Plant

\section{Introduction}

Palestine has a wide range of agro-ecological concerns and hosts a large variety of plants. Palestine's particular geographic location, in conjunction with a series of environmental and bioclimatic factors, makes this a very fertile land [1]. Climate and bioclimate factors played an important role in influence on plant communities and biological resources [2]. The flora of Palestine includes 149 endemic species (6\% of the total flora), of which $43 \%$ are found to be common; $27.5 \%$ are rare and $25.6 \%$ are very rare. Leguminaceae family for instance with its 268 species contains 21 endemics, while among 23 species of Iridaceae, eight are endemic [3], while today more than 155 endemic species. However, it is the meeting ground for plant species originating from wide-flung world regions, as far apart as Western Europe, Central Asia and Eastern Africa. It is characterized by a large variety of wildlife resources and represents a rich base of flora and fauna where the natural biota is composed by an estimated 2483 species of plants that inhabit Palestine [4]. Palestine, located in the Mediterranean basin, is considered as one of the world's biodiversity "hotspot" that should be subjected to conservation [5] [6]. Flora of Palestine is rich with economical important plants including vegetables, cereals and fruit trees, providing the local market with essential agricultural crops. Furthermore, more than 2750 species of plants including 138 families were estimated for Palestinian flora [7] [8].

The main aims of the present study are to contribute to the knowledge of the mean plant, and to phytosociological with others characteristics biologicals and ecologically of plant in Palestine.

\section{Materials and Methods}

Due to the lack of substantial volumes of meteorological data, we selected a sampling area in the region of Hebron, in which inventories were taken of 237 woody plants in the location of Idna. Forests and shrub lands were sampled in order to obtain biological indicators for thermicity and ombrotype; 237 samples of woody plant communities were selected as statistically significant number; although 237 inventories of herbaceous plants were also taken simultaneously in the same locations for subsequent studies. The inventories were made following Braun-Blanquet [9], and the Flora of Syria, Palestine and Sinai were used for the floristic study. The absence of phytosociological studies on the area led us to run a statistical treatment on the 237 woody plant inventories. One cluster analysis, Ward's method is applied, and the principal component analysis PCA is used to establish the different groups of plant communities, which has been used CAP3 software = Community Analysis Package III. Moreover, we transformed the Braun-Blanquet species abundance-dominance values into those of Van der Maarel [10]. We found only a few phytosociological works on areas in Egypt which have no relation with our communities [11] and others of an ecological nature [12] [13]. The vegetation was interpreted according to several methodological works [14]-[17].

\subsection{Study Area}

Idna (Idhna) is Palestinian town located to the north-west of the city of Hebron (13 kilometers west of Hebron) and in the southern West Bank, one of the territories occupied in 1967 and is located on the Green Line (Figure 1), an area now estimated at 17,000 hectares after it was 37,000 hectares in 1948, rises $500 \mathrm{~m}$ above sea level and between longitudes $34^{\circ} 06^{\prime}$ east and latitudes $31^{\circ} 33^{\prime}$ north.

Idna is physically divided into southern and northern parts by the Wadi Al-Feranj, and Wadi Risha and Beit Elban; western, Wadi Al-Balota and Al-Naqieh and primary source of income is agriculture and the town's total land area is 21,526 dunams $\left(215 \mathrm{~km}^{2}\right)$, of which 2809 dunams $\left(28 \mathrm{~km}^{2}\right)$ are built up area. Furthermore, the geographic location of Idna plays a major role in affecting the features of its climate and the biodiversity. They contain a biodiversity of plants such as trees, crops, vegetables $\&$ various wild plants.

In the location and physical characteristics, Idna is located on low altitude hills ranging from $400 \mathrm{~m}$ to $500 \mathrm{~m}$ above sea level with mean annual rainfall in Idna between 410 and 440 , average annual temperature of $19^{\circ} \mathrm{C}$ and 


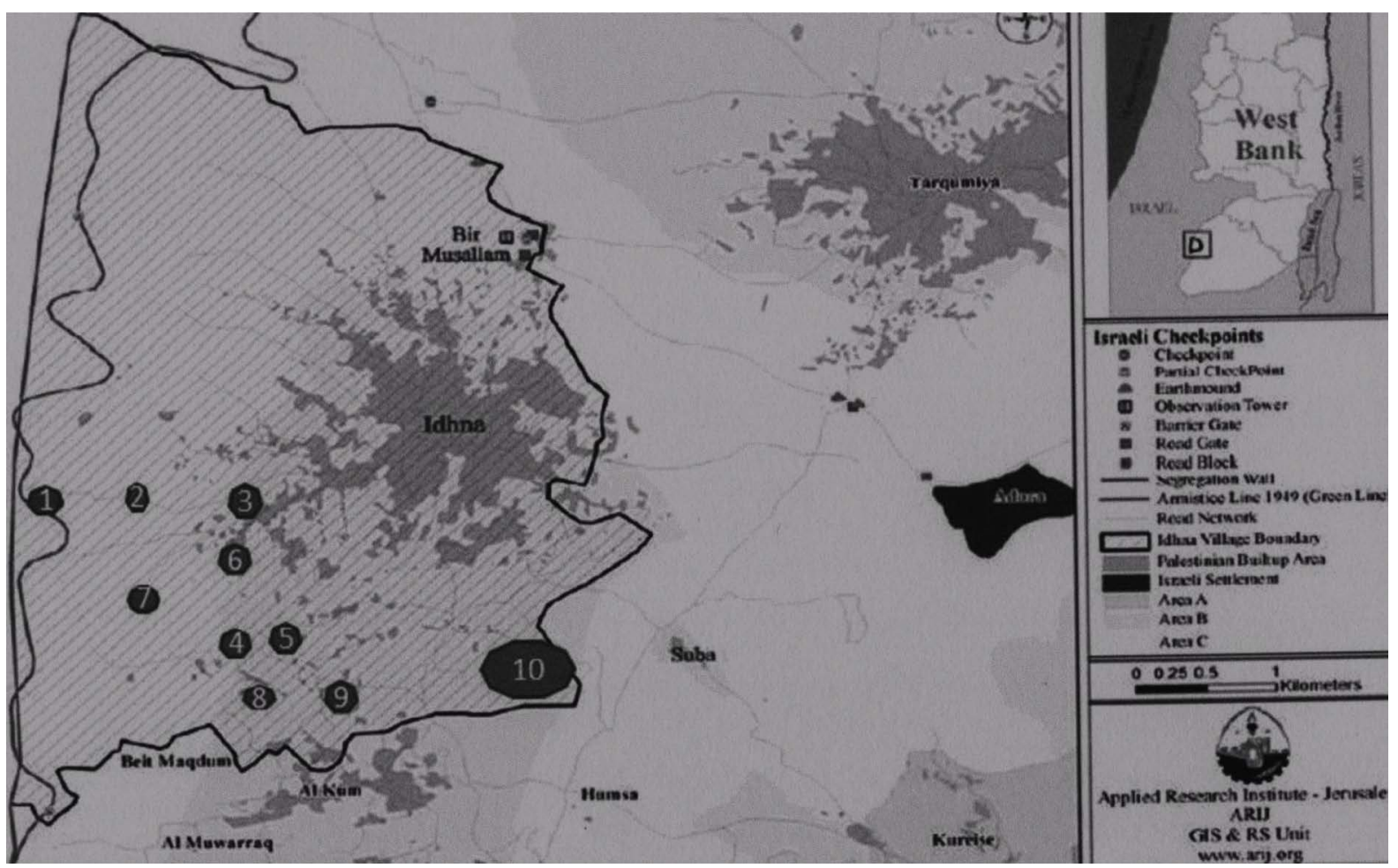

Figure 1. Map of Idna area showing different investigate locations and numbers represents a sampling place on the map.

the average annual humidity is about $60 \%$ [18].

\subsection{Targeting and Collection of Plant Materials}

We took 237 samples of different species plants from Idna village and selected samples study from Idna area such as (Wadi Al-Shumer \& Almrabeih, Khalit Al-Thora, Khalit Al-Khamga, Khalit Al-Karami, Shieb Ghazal, Alras, Wadi Al-Nagieh, Wadi Reshi, Ganan Jeash, Suba, and etc.) (Table 1).

\section{Results and Discussion}

\section{Vegetation Analysis}

Generally, today Palestine has nineteen principal plant communities such as savanna Mediterranean, Mediterranean, and sand, Maquis, Oak Woodlands and others [19]. In the statistical treatment we obtained two large groups in the cluster (Figure 2); group (A), representing forests, copses and high shrub lands influenced by climate (climatophilous); and group (B), representing Tamarix copses, which are thus edaphohygrophilic. For the aspect that concerns us here, there are six important plant communities in group A.

A1) Community association one (ASL1) comprises the inventories in cluster 1 - 183, and represents the forests of Quercus look with Pistacia palaestina, Ceratonia siliqua, Capparis decidua, Rhamnus punctata, Arbutus andrachne: forests growing in thermomediterranean dry-subhumid environments on carbonated substrates (terra rossaas brown rendizinas) with neutral $\mathrm{pH}$, which is demonstrated by the presence of Arbutus andrachne. This leads us to propose, for the Asiatic areas of the eastern Mediterranean, the association Pistacio palaestinaeQuercetum lokii (Table 2: ASL1_1-183).

A2) Community of Ceratonia siliqua, Capparis sinaica and the Asclepiadaceae of Sudanese origin Calotropis procera found growing in the stoniest and most thermophilous areas of the semiarid-dry inframediterranean. This is a high scrubland with a deserted semi-steppe character located to the east the region of Hebron, near the steppes of the Negev where the ombrotype is arid-semiarid, and the soil types are lithosoils and loess. The presence of Ceratonia siliqua and Pistacia palaestina in these communities is due to the presence of rock beds that retain humidity. For these thermophilous and semiarid areas we propose the association Capparido sinaicae- 


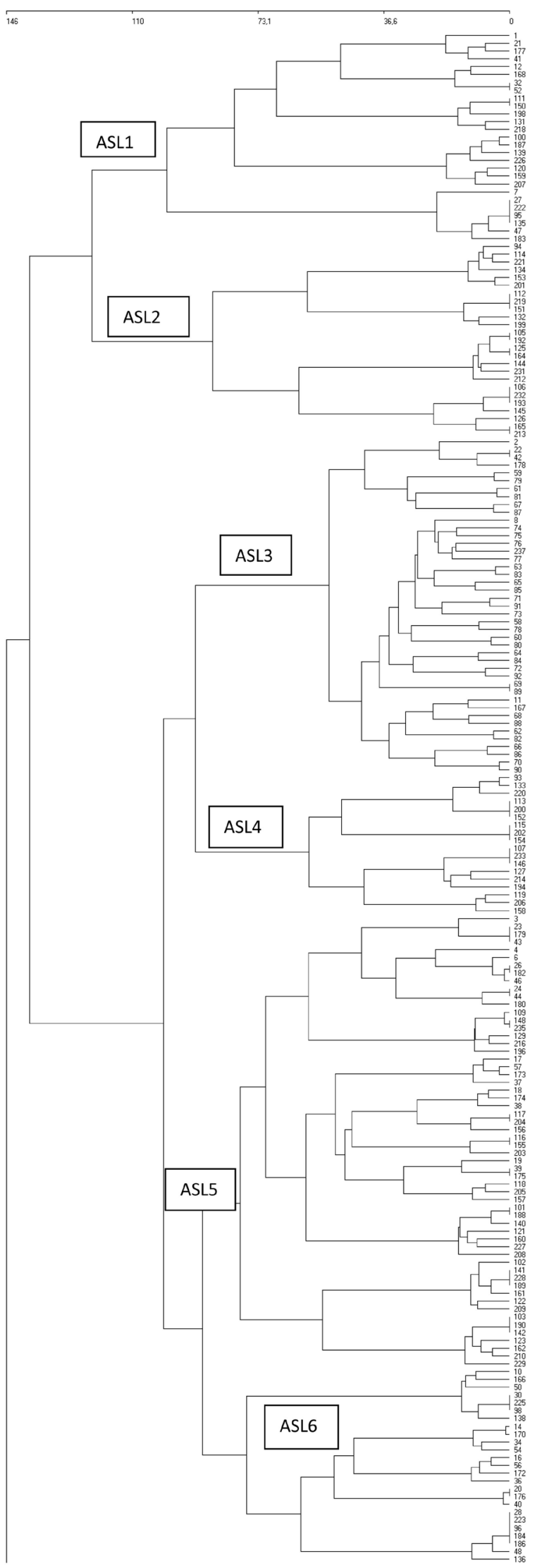




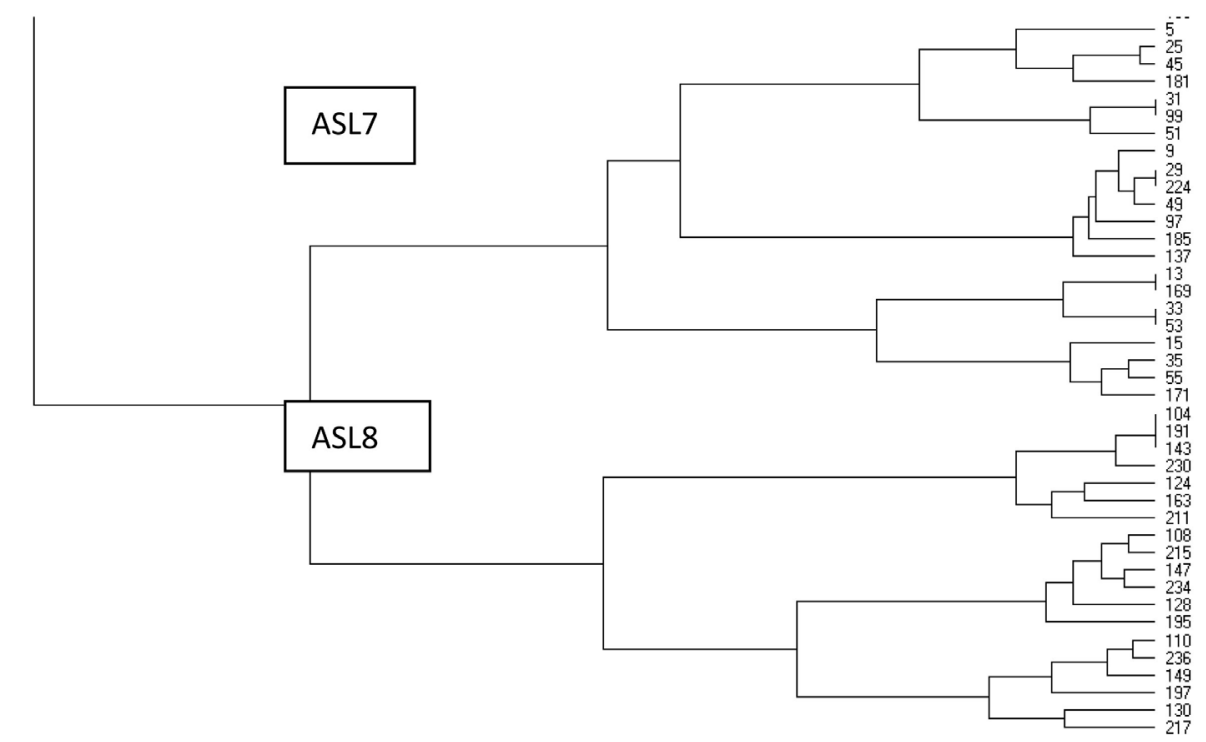

Figure 2. Cluster analysis of woody vegetation of Palestine.

Table 1. Details of the tenth stands chosen for vegetation analysis.

\begin{tabular}{cc} 
Number & Location \\
\hline $\mathbf{1}$ & Wadi Al-Shumer \& Almrabeih \\
$\mathbf{2}$ & Khalit Al-Thora \\
$\mathbf{4}$ & Khalit Al-Khamga \\
$\mathbf{5}$ & Khalit Al-Karami \\
$\mathbf{6}$ & Shieb Ghazal \\
$\mathbf{7}$ & Alras \\
$\mathbf{8}$ & Wadi Al-Nagieh \\
$\mathbf{9}$ & Wadi Reshi \\
$\mathbf{1 0}$ & Ganan Jeash \\
\hline
\end{tabular}

Ceratonietum siliquae (Table 3: ASL2_94-213).

A3) A marcescent forest of Quercus ithaburensis, Cerasus macrocarpa, Rhus penthylla and Pyrus siriaca found growing in the region of Hebron on calcareous of carbonated substrates in sub-humid-humid mountain environments in the thermo-mesomediterranean; its area of distribution is most likely the whole of the Palestinian mountains. For this we propose the association Cerasus microcarpae-Quercetum ithaburensis (Table 4: ASL3_2-90).

A4) An almost pure forest of Abies cilicica and Pyrus siriaca, together with other species such as Rhus pentaphylla, Rhus tripartite and Cerasus prostrate growing in the highest, rainiest and coldest areas of the mountains of (Hebron) on calcareous substrates in the humid mesomediterranean. This type of forest is accompanied in specific areas by the Lebanese cedar, and occasionally by deciduous elements such as Fagus crenata etc. We propose the association Pyro siriacae-Abietetum cilicicae (Table 5: ASL4_93-158).

A5) A mixed forest of Abies cilicica and Ceratonia siliqua, with introgression of more xeric elements such as Capparis decidua, Capparis sinaica, Peryploca aphylla and Pistacia lentiscos found in subhumid-humid and thermophilous areas in the thermomediterranean thermotype and rocky environments. We propose the association Abio ciliciae-Ceratonietum siliquae (Table 6: ASL5_3-229).

A6) Pine forest of Pinus halepensis, Periploca aphylla, Pistacia lentiscos, Rhamnus lycioides, Quercus calliprinos, a climatophilic community with an average degree of coverage, in semiarid-dry areas enriched with less xerophilous plants, growing in steppe environments with a semiarid-dry ombroclimate and an inframediterranean thermotype. We propose the association Periploco aphylli-Pinetum halepensis (Table 7: ASL6_10-136). 
Table 2. ASL1 from (1 - 183 of the cluster). Pistacio palestinae-Quercetum lokii Ighbareyeh, Cano-Ortiz \& Cano nova.

\begin{tabular}{|c|c|c|c|c|c|c|c|c|c|c|c|c|c|c|c|c|c|c|c|c|c|c|c|c|c|c|c|}
\hline a & 1 & & & & & 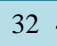 & 4 & & & & & & & & & & & & & & & & & & & & \\
\hline Altitu & 460 & & & & & 4705 & 501 & 435 & 461 & 573 & 5 & 6 & 6 & 6 & 600 & 463 & 454 & 513 & 418 & 4904 & 4904 & 468 & 416 & 515 & 4 & 3 & 45 \\
\hline Surfa & 80 & & 100 & 60 & 100 & 100 & 801 & 100 & 80 & 1 & 1 & 1 & 1 & 1 & 100 & 300 & 250 & 250 & 280 & 3003 & 3003 & 3002 & 250 & 3003 & 3003 & 0 & 250 \\
\hline Cov & 80 & 90 & 95 & 95 & 90 & 75 & 75 & 90 & 60 & 80 & 75 & 70 & 80 & 80 & 80 & 65 & 60 & 60 & 65 & 60 & 60 & 60 & 65 & 65 & 70 & 0 & 70 \\
\hline Avarage & 3 & 3 & 3 & 3 & 3 & 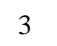 & 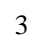 & 3 & 3.5 & 3.5 & 3.5 & 3.5 & 3.5 & 3.5 & 3.5 & 3 & 4 & 5 & 5 & 5 & 4 & 4 & 5 & 4 & 5 & & 4 \\
\hline Slo & 20 & 10 & 5 & 10 & 5 & 5 & 5 & 10 & 10 & 5 & 5 & 15 & 15 & 10 & 5 & 5 & 15 & 10 & 5 & 10 & 5 & 10 & 15 & 20 & 10 & 5 & 15 \\
\hline O & E & $\mathrm{N}$ & W & W & $\mathrm{N}$ & $\mathrm{W}$ & $\mathrm{N}$ & $\mathrm{E}$ & $\mathrm{N}$ & W & W & $\mathrm{N}$ & $\mathrm{S}$ & $\mathrm{S}$ & $\mathrm{E}$ & w & $\mathrm{N}$ & $\mathrm{N}$ & W & $\mathrm{N}$ & W & W & W & $\mathrm{S}$ & $\mathrm{F}$ & $\mathrm{F}$ & W \\
\hline \multicolumn{28}{|c|}{ haracteristic of the association and upper units } \\
\hline Pistacia palaestina Boiss. & 4 & 4 & 3 & 3 & 3 & 3 & 3 & 3 & 3 & 3 & 2 & 2 & 1 & 1 & 3 & 2 & 3 & 2 & 3 & 3 & 3 & 2 & 3 & 2 & 2 & 4 & 2 \\
\hline$Q$ & 3 & 3 & 3 & 3 & 4 & 2 & 3 & 4 & 3 & 4 & 3 & 3 & 3 & 3 & 4 & 3 & 3 & 3 & 2 & 3 & 3 & 2 & 3 & 3 & 3 & 4 & 2 \\
\hline Pinu & 2 & & 2 & 2 & & 2 & 2 & $\cdot$ & 2 & . & 2 & 2 & 2 & 2 & . & 2 & 2 & 2 & 2 & 2 & . & 2 & 2 & 2 & 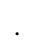 & & 2 \\
\hline C & . & 1 & 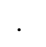 & . & 2 & & . & 2 & $\cdot$ & 2 & 3 & . & 3 & $\cdot$ & 2 & 3 & & 3 & 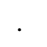 & . & 2 & 3 & 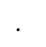 & 3 & & 2 & 3 \\
\hline $\mathrm{Cal}$ & . & & 2 & . & . & 2 & . & . & 2 & . & 2 & 2 & 2 & 2 & 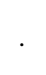 & 2 & 2 & 2 & 2 & . & & 2 & 2 & 2 & 2 & & 2 \\
\hline Bryonia & $\cdot$ & . & 2 & $\cdot$ & . & 2 & & . & 2 & $\cdot$ & $I$ & 2 & 1 & 2 & 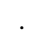 & 1 & 2 & 1 & $\cdot$ & . & . & 1 & 2 & 1 & 2 & & 1 \\
\hline Rham & 4 & ${ }^{\circ}$ & 2 & $\cdot$ & . & 2 & S & . & 2 & . & 3 & . & 3 & $\cdot$ & . & 3 & . & 3 & 2 & . & . & 3 & . & 5 & r. & & $\mathrm{S}$ \\
\hline Rhus & . & $\cdot$ & . & . & . & . & r. & . & . & . & 3 & 3 & 3 & 3 & . & 3 & 3 & 2 & . & . & . & 4 & . & 4 & 3 & r. & 4 \\
\hline A & . & $\cdot$ & 2 & . & . & 2 & . & . & 2 & . & . & 2 & . & 2 & . & . & 2 & . & 2 & . & 3 & . & 2 & . & 2 & 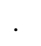 & 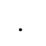 \\
\hline Periploca aph & 2 & . & 2 & 2 & . & 2 & 2 & . & 2 & . & . & 2 & . & 2 & . & . & 2 & . & 2 & 2 & . & . & 2 & . & 2 & . & 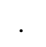 \\
\hline $\mathrm{L}$ & 3 & 3 & . & . & . & . & . & . & . & . & . & 2 & . & 2 & . & . & 2 & . & . & . & . & . & 2 & . & 2 & $\cdot$ & 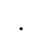 \\
\hline Pisto & 2 & 2 & 5 & . & 2 & . & 2 & 2 & . & 2 & . & . & . & . & 2 & • & . & . & . & . & 2 & . & . & . & . & 2 & \\
\hline$P i$ & . & . & 2 & . & . & 2 & . & . & 2 & . & . & 2 & . & 2 & . & . & 2 & . & 2 & . & . & . & 2 & . & 2 & . & \\
\hline & · & . & 2 & r. & . & 2 & . & . & 2 & . & . & . & . & . & . & . & . & . & 2 & • & . & . & . & . & & & \\
\hline$D$ & . & . & . & . & . & . & . & . & . & r & . & 2 & . & 2 & . & . & 2 & . & . & . & . & . & 2 & . & 2 & $\cdot$ & \\
\hline & 1 & $\cdot$ & 2 & 1 & $\cdot$ & 2 & 1 & . & 2 & . & 2 & $\cdot$ & 2 & . & $\cdot$ & . & . & . & . & 1 & . & 2 & $\cdot$ & 2 & & $\cdot$ & \\
\hline Cera & . & . & . & . & r & . & . & r & . & . & . & + & . & . & . & . & + & . & . & . & . & . & + & . & & . & \\
\hline Cerasus & . & + & + & . & • & . & & . & ${ }^{\circ}$ & . & . & $\cdot$ & · & $\cdot$ & . & . & $\cdot$ & $\cdot$ & + & . & $\cdot$ & . & . & . & & • & \\
\hline Rham & 2 & $\cdot$ & . & . & $\cdot$ & . & 2 & . & • & $\cdot$ & . & . & . & . & . & . & . & . & . & . & . & . & . & & & . & \\
\hline Pis & . & . & . & . & . & . & . & . & - & . & . & 2 & . & 2 & . & . & 2 & . & . & . & . & . & 2 & . & . & . & \\
\hline Querc & 2 & . & . & 2 & . & . & 2 & . & . & . & . & . & . & . & . & . & . & . & . & 2 & . & . & . & . & & . & \\
\hline & $\cdot$ & $\cdot$ & . & . & . & . & . & . & . & . & . & . & . & . & . & 1 & . & 3 & . & . & . & . & . & 3 & & . & \\
\hline$C \epsilon$ & . & + & . & . & . & . & . & . & . & . & . & . & . & . & . & . & . & . & . & . & . & . & . & . & . & . & \\
\hline Crataegus sinaicus Boiss. & . & . & . & . & . & . & . & . & & . & . & . & . & . & . & 1 & & . & . & . & . & . & . & . & . & . & \\
\hline
\end{tabular}

Finally in the cluster (B) group we differentiate two types of Tamarix copses; first those located in the western areas of the territory (B1) in gorges with intermittent saline or non-saline water in the inframediterranean-thermo-mediterranean thermotype where the dominant species are Tamarix tetragyna, Tamarix passerioides, and Tamarix palaestina. In this case the Tamarix copse includes the species Cytisopsis pseudocytisus. We propose the association Cytisopsis pseudocytiso-Tamaricetum tetragynae (Table 8: ASL7_5-171). The tamarisk grove in the more eastern areas of the territory, in the semiarid-dry infra-Mediterranean thermotype in highly saline gorges is dominated by Tamarix jordanis, Tamarix passerinoides and Tamarix palaestina accompanied by Capparis decidua, Capparis sinaica and Crataegus sinaicus. We propose the association Crataego sinaicae-Tamaricetum jordanii (Table 9: ASL8_104-217).

However, both associations of Tamarix should be included in the Mediterranean and Saharan-Arabian class Nerio-Tamaricetea [20]; and in Tamaricetalia [21] [22] (Braun-Blanquet J. (1952a, b)) the only order described to date, the absence of Tamarix gallica and Tamarix africana, and the presence in Asiatic territories of Tamarix tetragyna, T. tetrandra, T. jordanis etc., provisionally allows us to create the new alliance Tamaricion tetragynae. 
Table 3. ASL2 from (94 - 213 of the cluster). Capparido sinaicae-Ceratonietum siliquae Ighbareyeh, Cano-Ortiz \& Cano nova.

\begin{tabular}{|c|c|c|c|c|c|c|c|c|c|c|c|c|c|c|c|c|c|c|c|c|c|c|c|c|c|}
\hline r of cluster & 94 & & 1 & 1 & 1 & 1 & 126 & 132 & 134 & & 4145 & 151 & 153 & 164 & 165 & 192 & 193 & 199 & 201 & 212 & 213 & 219 & 221 & 231 & 232 \\
\hline Altitu & 570 & & & & & 640 & 633 & 617 & 500 & & 4403 & & 4 & 4 & & 4 & 426 & 449 & 454 & 455 & 465 & 3 & 434 & 480 & 5 \\
\hline Surfa & 100 & 10 & 10 & 10 & 10 & 10 & 100 & 100 & 100 & 2 & 300 & 300 & 300 & 250 & 300 & 300 & 300 & 280 & 250 & 300 & 300 & 280 & 300 & 300 & 300 \\
\hline Cover rate \% & 75 & 90 & 95 & 50 & 75 & 75 & 80 & 50 & 80 & 80 & 50 & 40 & 60 & 80 & 60 & 60 & 55 & 60 & 25 & 35 & 25 & 35 & 40 & 30 & 35 \\
\hline Avarage heig & 3.5 & 3.5 & 3.5 & 3.5 & 3.5 & 3.5 & 3.5 & 3.5 & 3.5 & 4 & 3 & 5 & 5 & 4 & 4 & 4 & 3 & 5 & 4 & 4 & 5 & 5 & 5 & 3 & 5 \\
\hline Slope \% & 15 & 5 & 15 & 5 & 5 & 5 & 5 & 5 & 5 & 10 & 15 & 10 & 5 & 10 & 5 & 18 & 20 & 8 & 15 & 15 & 5 & 5 & 15 & 5 & 15 \\
\hline Orient & S & $\mathrm{N}$ & $\mathrm{W}$ & $\mathrm{S}$ & $\mathrm{W}$ & $\mathrm{W}$ & $\mathrm{N}$ & $\mathrm{E}$ & $\mathrm{S}$ & $\mathrm{N}$ & $\mathrm{W}$ & S & $\mathrm{E}$ & $\mathrm{E}$ & $\mathrm{S}$ & $\mathrm{W}$ & $\mathrm{N}$ & $\mathrm{N}$ & $\mathrm{N}$ & $\mathrm{E}$ & $\mathrm{S}$ & $\mathrm{W}$ & $\mathrm{N}$ & $\mathrm{S}$ & $\mathrm{N}$ \\
\hline \multicolumn{26}{|c|}{ Characteristic of the association and upper units } \\
\hline Ceratonia siliqu & 3 & 4 & 4 & 2 & 2 & 3 & 4 & 2 & 4 & 4 & 2 & 2 & 2 & 4 & 3 & 4 & 2 & 3 & 2 & 4 & 3 & 3 & 3 & 4 & 1 \\
\hline Calotropis procera (Aiton) W. T. Aiton & 3 & 2 & 2 & . & 3 & 2 & 2 & . & 3 & 2 & 2 & 1 & 3 & 2 & 2 & 2 & 2 & 1 & 3 & 2 & 2 & 1 & 3 & 2 & 2 \\
\hline Capparis sinaica Denne & 1 & 2 & 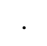 & 2 & $\cdot$ & 2 & • & 2 & 2 & 2 & . & 2 & 1 & . & . & 1 & $\cdot$ & 2 & + & . & . & 2 & 2 & . & . \\
\hline Pistacia palaestina Boiss. & 2 & $\cdot$ & 2 & ${ }^{\circ}$ & 2 & . & 2 & - & 2 & $\cdot$ & 2 & $\cdot$ & 2 & $\cdot$ & 2 & . & 2 & . & 2 & . & 2 & . & 2 & . & 2 \\
\hline $\begin{array}{c}\text { Abies cilicica (Antoine \& Koschy) } \\
\text { Carriere }\end{array}$ & 2 & . & 1 & . & + & . & 2 & . & 2 & . & 1 & . & + & . & 2 & . & 1 & . & + &. & 2 & . & 2 & . & 1 \\
\hline Rhus pentaphylla (Jacq.) Desf. & 2 & $\cdot$ & . & 2 & 2 & $\cdot$ & . & 2 & 2 & . & . & 2 & 2 & $\cdot$ & . & . & . & . & . & . & . & 2 & 2 & . & . \\
\hline Capparis decidua (Forssk.) Edgew. & . & . & 3 & ${ }^{\circ}$ & $\cdot$ & $\cdot$ & 3 & . & . & . & 3 & $\cdot$ & $\cdot$ & . & 3 & $\cdot$ & 3 & . & . & . & 3 & . & . & . & 3 \\
\hline Rhamnus lycioides Brot. & . & . & . & 2 & $\cdot$ & . & · & 2 & . & . & . & 2 & $\cdot$ & . & . & . & . & 2 & . & . & . & 2 & . & . & $\cdot$ \\
\hline Cerasus prostrata Labill & . & . & + & . & . & . & . & . & 4 & $\cdot$ & + & $\cdot$ & $\cdot$ & . & . & . & + & . & . & $\cdot$ & . & . & . & . & + \\
\hline $\begin{array}{c}\text { Cerasus microcarpa (C. A. Mey.) } \\
\text { Boiss. }\end{array}$ & . & $\cdot$ & $\cdot$ & . & 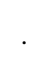 & $\cdot$ & 4 & 4 & $\cdot$ & $\cdot$ & 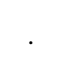 & $\cdot$ & $\cdot$ & $\cdot$ & $\cdot$ & $\cdot$ & $\cdot$ & $\cdot$ & $\cdot$ & $\cdot$ & $\cdot$ & $\cdot$ & $\cdot$ & $\cdot$ & $\cdot$ \\
\hline Quercus ithaburensi Kotschy & . & . & . & · & . & . & & . & . & . & 3 & $\cdot$ & . & . & . & . & . & . & . & . & . & . & . & $\cdot$ & . \\
\hline Bryonia dioica Jacq. & . & + & . & . & . & . & . & . & . & + & . & . & . & . & . & + & . & . & . & . & . & . & . & + & \\
\hline
\end{tabular}

Table 4. ASL3 from (2 - 90 of the cluster). Cerasus microcarpae-Quercetum ithaburensis Ighbareyeh, Cano-Ortiz \& Cano nova.

\begin{tabular}{|c|c|c|c|c|c|c|c|c|c|c|c|c|c|c|c|c|c|c|c|}
\hline Number of cluster & 2 & 78 & 79 & 80 & 81 & 82 & 83 & 84 & 85 & 86 & 87 & 88 & 89 & 90 & 91 & 92 & 167 & 178 & 237 \\
\hline Altitude in $\mathrm{m} 1=10$ & 485 & 483 & 494 & 487 & 457 & 462 & 467 & 512 & 511 & 539 & 525 & 514 & 522 & 535 & 531 & 537 & 420 & 480 & 479 \\
\hline Surface in $\mathbf{m}^{2}$ & 100 & 100 & 100 & 100 & 100 & 100 & 100 & 100 & 100 & 100 & 100 & 100 & 100 & 100 & 100 & 100 & 300 & 300 & 300 \\
\hline Cover rate \% & 70 & 90 & 80 & 95 & 95 & 90 & 60 & 65 & 60 & 75 & 75 & 80 & 85 & 60 & 65 & 65 & 45 & 35 & 35 \\
\hline Avarage height of veg. (m) & 4 & 4 & 5 & 3.5 & 3.5 & 3.5 & 4.5 & 4.5 & 4.5 & 4.5 & 4.5 & 4.5 & 4.5 & 4.5 & 3.5 & 3.5 & 5 & 4 & 5 \\
\hline Slope \% & 10 & 20 & 10 & 10 & 5 & 5 & 5 & 5 & 5 & 5 & 5 & 15 & 5 & 5 & 5 & 5 & 10 & 5 & 15 \\
\hline Orientation & $\mathrm{N}$ & W & $\mathrm{E}$ & $\mathrm{W}$ & $\mathrm{E}$ & $\mathrm{E}$ & $\mathrm{E}$ & $\mathrm{E}$ & $\mathrm{N}$ & $\mathrm{N}$ & $\mathrm{E}$ & S & S & $\mathrm{S}$ & $\mathrm{N}$ & $\mathrm{N}$ & $\mathrm{E}$ & W & $\mathrm{N}$ \\
\hline \multicolumn{20}{|c|}{ Characteristic of the association and upper units } \\
\hline Quercus ithaburensi Kotschy & 3 & 2 & 3 & 3 & 3 & 2 & 2 & 3 & 3 & 3 & 3 & 2 & 4 & 3 & 2 & 2 & 2 & 2 & 2 \\
\hline Cerasus microcarpa (C. A. Mey.) Boiss. & 2 & 2 & 3 & . & 2 & . & 2 & 1 & 3 & 1 & 2 & 3 & 2 & + & 2 & 2 & + & + & + \\
\hline Cerasus prostrata Labill. & 2 & 3 & 4 & 3 & 2 & 2 & 3 & 2 & 3 & 2 & 2 & . & . & . & 2 & 2 & . & 2 & + \\
\hline Rhus pentaphylla (Jacq.) Desf. & . & . & 3 & 3 & 2 & 4 & 3 & . & 2 & . & 2 & 2 & 2 & . & 2 & . & . & . & 1 \\
\hline Pyrus syriaca Boiss. & 2 & . & . & . & . & 2 & . & 2 & . & . & . & 2 & . & . & . & . & . & . & $\cdot$ \\
\hline Crataegus sinaicus Boiss. & 2 & . & . & . & . & 2 & . & . & . & . & . & 2 & . & . & . & . & . & . & . \\
\hline Rhamnus lycioides Brot. & 2 & 1 & 1 & 1 & . & 1 & . & . & . & . & 2 & . & . & 2 & . & 2 & . & . & . \\
\hline Arbutus andrachne L. & . & 2 & . & . & . & $\cdot$ & 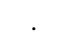 & 3 & . & . & . & . & 3 & . & 2 & 3 & . & . & . \\
\hline Pistacia lentiscus L. & . & . & . & . & . & 2 & . & . & 2 & . & . & . & 2 & . & 2 & . & . & . & 2 \\
\hline Quercus libani G. Olivier & . & 3 & . & 5 & . & . & . & . & 2 & . & . & . & . & . & . & . & . & . & . \\
\hline Rhamnus punctata Boiss. & . & 4 & 2 & . & . & & . & 2 & . & . & . & . & . & . & . & 2 & . & . & . \\
\hline
\end{tabular}




\section{Continued}

\begin{tabular}{|c|c|c|c|c|c|c|c|c|c|c|c|c|c|c|c|c|c|c|c|}
\hline Quercus calliprinos Webb. & 2 & . & . & . & . & . & . & 2 & 3 & . & . & . & . & . & 2 & 2 & & . & . \\
\hline Ceratonia siliqua L. & . & . & 4 & . & 4 & . & . & 3 & $\cdot$ & . & . & . & . & . & . & . & . & . & . \\
\hline Fagus crenata Blume & 1 & . & 2 & 2 & . & . & . & . & . & . & . & . & . & . & . & 1 & & . & . \\
\hline Pinus halepensis Mill. & . & . & . & . & . & . & . & . & . & 3 & . & . & 2 & . & . & . & & 2 & 2 \\
\hline Periploca aphylla Tourn ex. L. & . & . & . & . & . & . & . & . & . & . & 2 & . & . & . & 2 & . & & . & 2 \\
\hline Pistacia saportae Burnat & 2 & . & . & . & . & . & . & . & . & . & . & . & . & 2 & . & . & 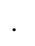 & . & . \\
\hline Rhus coriaria L. & 2 & . & 2 & . & . & 2 & . & . & . & . & . & 2 & . & . & . & 3 & . & . & . \\
\hline Celtis australi L. & 2 & . & . & . & . & $\cdot$ & . & . & . & . & . & 2 & . & . & . & . & . & . & . \\
\hline Calotropis procera (Aiton) W. T. Aiton & $\cdot$ & . & . & . & . & 2 & 2 & . & . & 2 & . & 2 & . & 3 & . & 2 & . & . & 2 \\
\hline Bryonia dioica Jacq. & . & 2 & . & . & . & . & . & . & . & . & . & . & . & . & . & . & . & . & . \\
\hline Capparis sinaica Denne & . & 1 & 1 & . & 1 & . & 1 & . & 1 & . & 1 & . & . & . & . & . & . & . & $\cdot$ \\
\hline Capparis decidua (Forssk.) Edgew. & . & . & . & . & 4 & . & . & 2 & . & . & . & . & . & . & . & 2 & . & . & . \\
\hline Rhus tripartita L. & . & . & . & . & . & . & . & 2 & . & . & . & $\cdot$ & . & . & . & . & . & . & . \\
\hline Bryonia syriaca Boiss. & . & $\cdot$ & . & 2 & . & . & . & . & . & . & . & . & . & . & + & + & . & . & . \\
\hline Quercus look Kotschy & . & $\cdot$ & . & . & . & . & . & . & . & . & . & . & . & . & . & . & 2 & . & . \\
\hline Abies cilicica (Antoine \& Koschy) Carriere & . & . & . & . & . & . & . & . & . & . & 1 & . & . & . & . & . & . & 1 & . \\
\hline Pistacia palaestina Boiss. & . & . & . & . & . & . & . & . & . & $\cdot$ & $\cdot$ & $\cdot$ & . & . & . & . & . & . & . \\
\hline Rhus tripartita L. & $\cdot$ & . & . & . & . & . & . & . & . & . & . & . & . & . & . & . & . & . & . \\
\hline Cercis siliquastrum L. & . & . & . & . & . & . & . & . & . & $\cdot$ & $\cdot$ & . & $\cdot$ & . & $\cdot$ & . & $\cdot$ & . & . \\
\hline Pistacia atlantica Desf. & . & . & . & . & . & . & . & . & . & . & . & . & . & . & . & . & . & . & . \\
\hline
\end{tabular}

Table 5. ASL4 from (93 - 158 of the cluster). Pyro siriacae-Abietetum cilicicae Ighbareyeh, Cano-Ortiz \& Cano nova.

\begin{tabular}{|c|c|c|c|c|c|c|c|c|c|c|c|c|c|c|c|c|c|c|}
\hline Number of cluster & 93 & 107 & 113 & 115 & 119 & 127 & 133 & 146 & 152 & 154 & 158 & 194 & 200 & 202 & 206 & 214 & 220 & 233 \\
\hline Altitude in $\mathbf{m}$ & 535 & 570 & 618 & 602 & 580 & 620 & 574 & 385 & 406 & 466 & 505 & 466 & 444 & 534 & 499 & 452 & 431 & 500 \\
\hline Surface in $\mathbf{m}^{2}$ & 100 & 100 & 100 & 100 & 100 & 100 & 100 & 300 & 300 & 300 & 300 & 300 & 300 & 300 & 250 & 260 & 300 & 300 \\
\hline Cover rate \% & 60 & 60 & 60 & 60 & 50 & 80 & 70 & 60 & 40 & 45 & 60 & 40 & 30 & 65 & 60 & 60 & 40 & 65 \\
\hline Avarage height of veg. (m) & 2.5 & 2.5 & 2.5 & 2.5 & 2.5 & 2.5 & 2.5 & 4 & 4 & 5 & 4 & 5 & 5 & 5 & 5 & 4 & 4 & 5 \\
\hline Slope \% & 5 & 2.5 & 5 & 5 & 2.5 & 2.5 & 5 & 10 & 16 & 5 & 10 & 5 & 10 & 10 & 5 & 10 & 10 & 10 \\
\hline Orientation & $\mathrm{N}$ & $\mathrm{N}$ & $\mathrm{N}$ & $\mathrm{N}$ & $\mathrm{S}$ & W & $\mathrm{W}$ & $\mathrm{N}$ & $\mathrm{E}$ & $\mathrm{S}$ & $\mathrm{S}$ & $\mathrm{N}$ & $\mathrm{N}$ & $\mathrm{S}$ & $\mathrm{E}$ & W & $\mathrm{W}$ & $\mathrm{N}$ \\
\hline \multicolumn{19}{|l|}{ Characteristic of the association and upper units } \\
\hline Abies cilicica (Antoine \& Koschy) Carriere & 2 & 3 & 3 & 3 & 2 & 4 & 3 & 3 & 2 & 2 & 3 & 3 & 2 & 2 & 3 & 3 & 2 & 3 \\
\hline Ceratonia siliqua L. & 2 & 3 & + & 1 & . & 3 & 2 & 3 & + & 1 & . & 3 & + & 1 & . & 3 & 2 & 3 \\
\hline Rhus pentaphylla (Jacq.) Desf. & 2 & 2 & 2 & 3 & 2 & 2 & 2 & 2 & 2 & 3 & 2 & 2 & 2 & 3 & 2 & 2 & 2 & 2 \\
\hline Rhus tripartita Lobadium Raf. & 2 & 2 & 2 & 2 & 2 & 2 & 2 & 2 & 2 & 2 & 2 & 2 & 2 & 2 & 2 & 2 & 2 & 2 \\
\hline Pyrus syriaca Boiss. & 1 & . & . & . & 2 & . & . & 1 & . & . & 2 & . & 1 & . & 2 & . & . & . \\
\hline Rhamnus lycioides Brot. & . & . & . & + & 2 & 3 & . & . & . & + & 2 & . & . & + & 2 & . & . & . \\
\hline Cerasus prostrata Labill. & . & 1 & . & . & 2 & 3 & . & 1 & . & . & 2 & 1 & . & . & 2 & . & . & 1 \\
\hline Capparis decidua (Forssk.) Edgew & 2 & . & 2 & . & . & . & 2 & . & 2 & . & . & . & 2 & . & . & . & . & . \\
\hline Quercus look Kotschy & 2 & . & 2 & . & . & . & 2 & . & 2 & . & . & . & 2 & . & . & . & . & . \\
\hline Quercus libani G. Olivier & 2 & . & 2 & . & . & . & 2 & . & 2 & . & . & . & 2 & . & . & . & . & . \\
\hline Pinus halepensis Mill. & . & . & . & 2 & . & . & . & . & . & 2 & . & . & . & 2 & . & . & . & . \\
\hline Cupressus sempervirens L. & . & . & . & 2 & . & . & . & . & . & 2 & . & . & . & 2 & . & . & . & . \\
\hline Cerasus microcarpa (C. A. Mey.) Boiss. & . & . & . & . & 2 & . & . & . & . & . & 2 & . & . & . & 2 & . & . & . \\
\hline Rhus coriaria L. & . & + & . & . & . & . & . & + & . & . & . & + & . & . & . & . & . & + \\
\hline Bryonia dioica Jacq. & . & . & . & . & 2 & . & . & . & . & . & 2 & . & . & . & 2 & . & . & . \\
\hline Quercus ithaburensi Kotschy & . & . & . & . & . & 4 & . & . & . & . & . & . & . & . & . & . & . & . \\
\hline Ficus carica L. Sp. & . & 2 & . & . & . & 2 & . & 2 & . & . & . & 2 & . & . & . & 2 & . & 2 \\
\hline Pistacia atlantica Desf. & . & . & . & 2 & . & . & . & . & . & 2 & 3 & . & . & 2 & . & . & . & . \\
\hline Morus nigra L. & . & . & . & 2 & . & . & . & . & . & 2 & . & 3 & . & 2 & . & . & . & . \\
\hline Rubus fruticosus L. & . & . & . & 2 & . & . & . & . & . & 2 & . & . & . & 2 & . & . & . & . \\
\hline Rubus idaeus L. & . & . & . & . & . & . & 1 & . & . & . & 3 & . & . & . & . & . & . & . \\
\hline
\end{tabular}


Table 6. ASL5 from (3 - 229 of the cluster). Abio cilicicae-Ceratonietum siliquae Ighbareyeh, Cano-Ortiz \& Cano nova (table with a number of part inventories cluster).

\begin{tabular}{|c|c|c|c|c|c|c|c|c|c|c|c|c|c|c|c|c|c|c|c|c|c|c|c|c|c|c|c|c|}
\hline Number of cluster & 3 & 4 & 6 & 17 & 18 & 19 & 23 & 24 & 26 & 37 & 38 & 39 & 43 & 44 & 46 & 57 & 101 & 102 & 103 & 109 & 9117 & 118 & 121 & 122 & 129 & 140 & 141 & 1148 \\
\hline Altitude in $\mathrm{m}$ & 450 & 439 & 429 & 462 & 469 & 445 & 417 & 420 & 408 & 493 & 475 & 500 & 479 & 485 & 434 & 421 & 580 & 570 & 600 & 596 & 6599 & 588 & 3577 & 7559 & 597 & 7466 & 453 & 3430 \\
\hline Surface in $\mathbf{m}^{2}$ & 80 & 100 & 80 & 100 & 100 & 100 & 100 & 100 & 100 & 100 & 100 & 100 & 100 & 100 & 100 & 100 & 100 & 100 & 100 & 100 & 0100 & 100 & 100 & 100 & 100 & 300 & 300 & 0280 \\
\hline Cover rate \% & 90 & 75 & 80 & 85 & 80 & 90 & 50 & 90 & 60 & 80 & 50 & 75 & 75 & 80 & 65 & 65 & 80 & 60 & 80 & 50 & 60 & 75 & 80 & 70 & 75 & 80 & 65 & 50 \\
\hline Avarage height of veg. (m) & 4 & 4 & 4 & 4 & 4 & 4 & 4 & 5 & 5 & 4.5 & 4.5 & 5 & 3.4 & 3.5 & 4 & 4 & 4 & 4 & 4.5 & 4.5 & 5 & 5 & 5 & 4 & 4 & 4 & 4 & 5 \\
\hline Slope \% & 10 & 30 & 5 & 20 & 10 & 5 & 5 & 5 & 5 & 10 & 15 & 5 & 10 & 15 & 5 & 5 & 15 & 5 & 5 & 15 & 15 & 5 & 5 & 15 & 5 & 10 & 18 & 8 \\
\hline Orientation & $\mathrm{N}$ & $\mathrm{N}$ & $\mathrm{E}$ & $\mathrm{E}$ & $\mathrm{E}$ & $\mathrm{E}$ & W & $\mathrm{W}$ & $\mathrm{N}$ & $\mathrm{N}$ & $\mathrm{E}$ & $\mathrm{N}$ & $\mathrm{W}$ & $\mathrm{N}$ & W & $\mathrm{W}$ & $\mathrm{N}$ & $\mathrm{S}$ & $\mathrm{N}$ & $\mathrm{S}$ & $\mathrm{N}$ & $\mathrm{W}$ & $\mathrm{S}$ & $\mathrm{E}$ & $\mathrm{E}$ & $\mathrm{N}$ & $\mathrm{w}$ & $\mathrm{N}$ \\
\hline \multicolumn{29}{|c|}{ Characteristic of the association and upper units } \\
\hline Ceratonia siliqua L. & 3 & 3 & 3 & 4 & 3 & 3 & 2 & 3 & 3 & 4 & 2 & 3 & 3 & 3 & 3 & 3 & 4 & 3 & 4 & 2 & 2 & 3 & 4 & 3 & 2 & 4 & 3 & 2 \\
\hline Capparis sinaica Denne & 2 & 1 & 2 & 1 & 2 & . & 1 & . & 1 & 1 & $\cdot$ & $\cdot$ & . & . & . & . & 1 & 1 & . & 2 & 2 & 3 & 1 & 1 & 2 & 1 & 1 & 2 \\
\hline $\begin{array}{l}\text { Abies cilicica (Antoine \& } \\
\text { Koschy) Carriere }\end{array}$ & 1 & . & . & . & 2 & 2 & 1 & . & . & . & 2 & 3 & 1 & . & . & . & . & . & . & . & 2 & 3 & . & . & . & . & . & . \\
\hline Ficus carica L. & 1 & 2 & 1 & $\cdot$ & . & . & 1 & 2 & 1 & $\cdot$ & . & . & 3 & 2 & 3 & 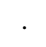 & . & . & . & . & 1 & . & . & . & . & . & . & . \\
\hline Periploca aphylla Tourn ex. L. & . . & 2 & . & . & . & . & . & 2 & . & + & + & $\cdot$ & . & 2 & ${ }^{\circ}$ & . & 2 & . & ${ }^{\circ}$ & . & . & . & 2 & . & . & 2 & . & . \\
\hline $\begin{array}{c}\text { Capparis decidua (Forssk.) } \\
\text { Edgew. }\end{array}$ & 1 & . & . & . & . & . & 1 & . & . & . & . & . & 1 & . & . & . & . & 2 & . & . & . & . & . & 2 & . & . & 2 & . \\
\hline Rhamnus punctata Boiss & . & 1 & . & . & . & . & . & . & . & . & . & . & . & . & . & . & . & . & 1 & • & . & . & . & . & . & . & . & . \\
\hline Bryonia syriaca Boiss. & + & + & + & . & . & . & . & . & $\cdot$ & . & . & . & . & . & . & . & . & . & . & 1 & . & . & . & . & 1 & 2 & . & 2 \\
\hline Rhamnus lycioides Brot. & . & 1 & . & . & . & . & . & . & . & . & . & . & . & . & . & . & . & . & . & . & 2 & . & 4 & . & . & . & . & . \\
\hline Quercus calliprinos Webb. & . & . & . & . & 2 & . & . & . & . & . & 2 & . & . & . & . & . & . & . & . & . & . & . & . & . & . & . & . & . \\
\hline Cerasus prostrata Labill & . & . & . & . & . & . & . & . & . & . & . & . & . & . & . & . & . & . & . & 1 & . & . & . & . & 3 & . & . & 1 \\
\hline Bryonia dioica Jacq. & . & . & . & . & . & . & . & . & . & . & . & . & . & . & . & . & + & + & + & . & . & . & . & . & . & + & + & . \\
\hline Rhus pentaphylla (Jacq.) Desf. & · & . & . & . & . & . & . & . & . & . & . & . & . & . & $\cdot$ & . & . & . & $\cdot$ & . & 2 & . & ${ }^{\circ}$ & . & . & . & . & . \\
\hline $\begin{array}{c}\text { Prunus dulcis (Mill.) D. A. } \\
\text { Webb. }\end{array}$ & . & . & . & 2 & 2 & . & . & . & . & 2 & 2 & . & . & . & . & 2 & . & . & . & 1 & . & . & . & . & . & . & . & 1 \\
\hline Pistacia palaestina Boiss. & . & . & . & . & . & . & . & . & . & . & . & . & . & . & . & . & . & . & 2 & . & . & . & . & . & . & . & . & . \\
\hline Pistacia saportae Burnat & . & 3 & . & . & . & . & . & 4 & . & . & . & . & . & 4 & . & . & . & . & . & . & . & . & . & . & . & . & . & . \\
\hline Pistacia atlantica Desf. & . & 3 & . & . & . & . & . & . & $\cdot$ & $\cdot$ & $\cdot$ & . & . & $\cdot$ & · & . & . & . & $\cdot$ & . & . & . & . & . & . & . & $\cdot$ & . \\
\hline Rhus tripartita L. & 4 & . & . & . & . & . & . & . & . & . & . & . & . & . & . & . & . & . & . & 3 & . & . & . & . & 3 & . & . & 3 \\
\hline Arbutus andrachne L. & . & . & . & . & . & . & . & . & . & . & . & · & . & . & . & . & . & 1 & . & . & . & . & . & 1 & . & . & 1 & . \\
\hline Quercus look Kotschy & 2 & . & . & 3 & . & . & 2 & . & . & 3 & . & . & 2 & . & . & 3 & . & . & . & . & . & . & . & . & . & . & . & . \\
\hline Quercus libani G. Olivier & . & . & . & 2 & . & . & . & . & . & 2 & . & . & . & . & . & 2 & . & . & . & . & . & . & . & . & . & . & . & . \\
\hline Pistacia lentiscus L. & 4 & . & . & . & . & 3 & . & . & . & + & + & 3 & 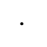 & • & $\cdot$ & • & . & · & . & . & . & . & . & . & . & . & . & . \\
\hline Pinus halepensis Mill. & . & . & . & . & . & . & . & . & . & + & + & · & . & . & . & . & . & . & 2 & . & . & . & . & . & . & . & . & . \\
\hline Cupressus sempervirens L. & . & . & . & . & . & . & . & . & . & + & + & . & . & . & . & . & . & . & . & . & 2 & . & . & . & . & . & . & . \\
\hline Pyrus syriaca Boiss. & . & . & . & . & . & . & . & . & . & . & . & . & . & . & . & . & . & . & . & . & . & . & 1 & . & . & . & . & . \\
\hline Rhus coriaria L. & . & . & . & 2 & . & . & . & . & . & . & . & . & . & . & . & . & . & . & . & . & . & . & . & 1 & . & . & . & . \\
\hline Celtis australi L. & . & . & + & . & . & . & . & . & . & . & . & . & . & . & . & . & . & . & . & . & . & . & . & . & . & . & . & . \\
\hline $\begin{array}{c}\text { Calotropis procera (Aiton) W. } \\
\text { T. Aiton }\end{array}$ & - 2 & . & 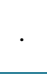 & - & . & . & 2 & . & . & . & . & ${ }^{\circ}$ & 2 & . & . & . & . & . & . & . & . & . & . & . & . & . & - & . \\
\hline
\end{tabular}

Furthermore, the six associations of copses and high shrub lands that represent sclerophyllous and marcescent vegetation in environments from the semiarid to the humid and from the thermo-supramediterranean are included in the class Quercetea ilicis [23]-[25]. However, the uncertainty as to the presence of Quercus ilex and Quercus coccifera, which is not manifested in the Flora of Palestine-although these species are found in the Flora of Palestine, Syria, Lebanon, Jordan, the Sinai-and the presence in these Asiatic territories of Quercus calliprinos, Quercus ithaburensis, Quercus libani and Quercus look obliges us to re-examine at least the alliance 
Table 7. ASL6 from (10 - 136 of the cluster). Periploco aphilli-Pinetum halepensi Ighbareyeh, Cano-Ortiz \& Cano nova.

\begin{tabular}{|c|c|c|c|c|c|c|c|c|c|c|c|c|c|c|c|c|c|c|c|c|c|c|c|c|c|}
\hline Number of cluster & 10 & 14 & 16 & 20 & 28 & 30 & 34 & 36 & 40 & 48 & 50 & 54 & 56 & 96 & 98 & & & & & $1 / 2$ & ) & & & & 322 \\
\hline Altitude in $\mathrm{m} 1=10$ & 471 & 430 & 466 & 392 & 435 & 445 & 480 & 492 & 495 & 429 & $420<$ & 4424 & 404 & 596 & 560 & & & & & & 495 & 475 & & & 440 \\
\hline Surface in $\mathbf{m}^{2}$ & 100 & 75 & 80 & 70 & 70 & 100 & 100 & 70 & 100 & 100 & 1001 & 1001 & 100 & 100 & 100 & 300 & 200 & 300 & 300 & 290 & 300 & 300 & 300 & 290 & 250 \\
\hline Cover $r$ & 80 & 85 & 80 & 95 & 75 & 80 & 70 & 95 & 85 & 80 & 85 & 85 & 85 & 60 & 80 & 35 & 80 & 80 & 60 & 80 & 70 & 60 & 60 & 60 & 60 \\
\hline Avarage hei & 1.5 & 1.5 & 1 & 1 & 1 & 1.5 & 1 & 1 & 1.5 & 1.5 & 1.5 & 1 & 1.5 & 1.5 & 1.5 & 4 & 4 & 5 & 5 & 5 & 4 & 4 & 5 & 5 & 5 \\
\hline c & 15 & 15 & 30 & 5 & 5 & 15 & 10 & 5 & 5 & 5 & 15 & 5 & 5 & 15 & 5 & 10 & 10 & 10 & 15 & 10 & 5 & 15 & 15 & 10 & 10 \\
\hline Orie & E & W & $\mathrm{N}$ & $\mathrm{W}$ & $\mathrm{N}$ & $\mathrm{W}$ & W & $\mathrm{W}$ & $\mathrm{W}$ & E & $\mathrm{E}$ & $\mathrm{W}$ & $\mathrm{E}$ & S & $\mathrm{N}$ & $\mathrm{W}$ & $\mathrm{W}$ & $\mathrm{S}$ & $\mathrm{N}$ & $\mathrm{N}$ & $\mathrm{E}$ & W & $\mathrm{N}$ & $\mathrm{N}$ & $\mathrm{N}$ \\
\hline \multicolumn{26}{|c|}{ Characteristic of the association and upper units } \\
\hline Pinus halepensis Mill. & 4 & 3 & 2 & 2 & 3 & 4 & 3 & 3 & 2 & 3 & 4 & 3 & 3 & 2 & 4 & 3 & 4 & 4 & 3 & 2 & 2 & 2 & 2 & 2 & 4 \\
\hline Pistacia & 2 & 2 & 2 & 3 & 2 & 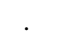 & 2 & + & 3 & $\cdot$ & 1 & 2 & 2 & 1 & . & 1 & ${ }^{\circ}$ & 1 & 2 & 2 & 3 & . & $\cdot$ & . & . \\
\hline Periploca ap & 1 & . & 2 & . & 1 & $\cdot$ & $\cdot$ & 1 & & $\cdot$ & . & 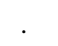 & 2 & & $\cdot$ & 1 & . & 1 & . & 2 & r. & . & 1 & 1 & . \\
\hline Rhus tripartita L. & 2 & 2 & 2 & . & . & 2 & 2 & 2 & & . & 2 & 2 & 2 & . & 2 & . & 2 & 2 & 2 & 2 & . & . & . & . & 2 \\
\hline Cupressus sempervirens L. & . & . & 4 & . & . & . & . & + & . & . & . & . & 4 & . & . & . & . & . & . & 4 & . & . & . & . & . \\
\hline Rhamnus punctata Boiss & . & . & 3 & . & . & . & . & 3 & . & . & . & . & 3 & & . & . & . & . & - & 3 & . & . & . & . & \\
\hline Rhamnus lyci & $\cdot$ & . & . & 2 & . & . & . & . & 2 & - & . & . & . & . & . & . & . & . & . & . & 2 & . & . & . & . \\
\hline Quercus calliprinos Webb. & 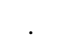 & . & + & 2 & 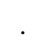 & 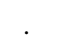 & . & . & 2 & 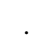 & 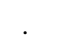 & 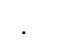 & & & . & . & . & . & & + & 2 & . & 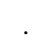 & . & \\
\hline
\end{tabular}

Table 8. ASL7 from (5 - 171 of the cluster). Cytisopsis pseudocytiso-Tamaricetum tetragynae Ighbareyeh, Cano-Ortiz \& Cano nova.

\begin{tabular}{|c|c|c|c|c|c|c|c|c|c|c|c|c|c|c|c|c|c|c|c|c|c|}
\hline \multirow{2}{*}{$\begin{array}{l}\text { Numero of cluster } \\
\text { Altitude in } \mathrm{m} 1=10\end{array}$} & 5 & 9 & 13 & 15 & 25 & 29 & 31 & 33 & 35 & 45 & 51 & 53 & 55 & 97 & \multicolumn{7}{|c|}{99137169171181185224} \\
\hline & 392 & 466 & 429 & 435 & 411 & 430 & 460 & 489 & 476 & 443 & 439 & 452 & 420 & 620 & 540 & 488 & 431 & 469 & 488 & 470 & 422 \\
\hline Surface in $\mathbf{m}^{2}$ & 80 & 100 & 60 & 80 & 100 & 100 & 100 & 100 & 100 & 100 & 100 & 100 & 100 & 100 & 100 & 250 & 300 & 300 & 300 & 300 & 300 \\
\hline Cover rate \% & 60 & 65 & 70 & 70 & 70 & 75 & 70 & 65 & 70 & 80 & 75 & 65 & 60 & 70 & 75 & 60 & 65 & 60 & 75 & 65 & 65 \\
\hline Avarage height of veg. (m) & 2 & 2 & 2 & 2.5 & 2.5 & 3 & 3 & 3 & 3 & 3 & 3 & 3 & 3 & 3.5 & 3.5 & 4 & 4 & 5 & 5 & 3 & 4 \\
\hline Slope \% & 5 & 5 & 10 & 10 & 5 & 10 & 10 & 5 & 5 & 5 & 10 & 5 & 10 & 15 & 15 & 15 & 10 & 5 & 15 & 5 & 5 \\
\hline Orientation & $\mathrm{S}$ & $\mathrm{E}$ & $\mathrm{E}$ & $\mathrm{E}$ & $\mathrm{N}$ & $\mathrm{E}$ & $\mathrm{E}$ & $\mathrm{N}$ & $\mathrm{S}$ & $\mathrm{E}$ & W & $\mathrm{W}$ & $\mathrm{N}$ & $\mathrm{N}$ & W & $\mathrm{W}$ & W & $\mathrm{E}$ & $\mathrm{N}$ & $\mathrm{S}$ & $\mathrm{W}$ \\
\hline \multicolumn{22}{|l|}{ Characteristic of the association and upper units } \\
\hline Tamarix tetragyna Ehrenb. & 3 & 3 & 3 & 3 & 3 & 3 & 3 & 3 & 3 & 3 & 3 & 3 & 3 & 3 & 3 & 3 & 3 & 3 & 3 & 3 & 3 \\
\hline Tamarix passerinoides Delile ex Desv. & 2 & 2 & 3 & 2 & 2 & 2 & 2 & 3 & 2 & 2 & 2 & 3 & 2 & 2 & 2 & 2 & 3 & 2 & 2 & 2 & 2 \\
\hline Cytisopsis pseudocytisus (Boiss.) Fertig & 3 & 3 & 2 & 3 & 3 & 3 & 2 & 2 & 3 & 3 & 2 & 2 & 3 & 3 & 2 & 3 & 2 & 3 & 3 & 3 & 3 \\
\hline Tamarix jordanis L. & 2 & . & . & . & 2 & . & 2 & . & . & 2 & 2 & . & . & . & 2 & 1 & . & . & 2 & . & . \\
\hline Tamarix palaestina Bertol & 3 & + & . & . & 3 & . & 3 & . & . & 3 & 3 & . & . & . & 3 & . & . & . & 3 & . & . \\
\hline Tamarix parviflora DC. & 2 & . & . & . & 2 & . & . & . & . & 2 & . & . & . & . & . & . & . & . & 2 & . & . \\
\hline
\end{tabular}

in which this type of communities should be included, which is currently under study. Also, distribution Atlas or plants the flora Palestinian area [7] comprises updated nomenclature, distribution and habit data for the species in the area covered by flora Palestinian [26]-[31]. However, the high frequency of Ceratonia siliqua, Pistacia lentiscus, Pinus halepensis, Olea sylvestris and Rhamnus lycioides allows us provisionally to include the communities in the most xeric environments in the order Pistacio-Rhamnetalia alaterni [32], and in the alliance Oleo-Ceratonión siliquae Br.-Bl. ex Guinochet \& Drouineau 1944, while we include the forests growing in dry, sub-humid environments in the order Quercetalia ilicis Br.-Bl. ex Molinier [33]-[35]. It is probably necessary to create a new alliance to include all these sclerophyllous and marcesent forests. Some differences between the different associations proposed can be seen in the synthetic table (Table 10: ASL9). However, we noted that the plants in Palestine are considered an important part of the plant to the Region Mediterranean basin, which is similar to many studies such as [19] [36]. Nevertheless, there coexistence between plants groups due to the bio-diversity and the topography, the nature of the climate and climatic factors and various other factors, while it's not found in some plant communities (Figure 3).

\section{Conclusions}

Palestine's geographical position has been both its blessing and its curse, located at the meeting point between 
Table 9. ASL8 from (104 - 217 of the cluster). Crateago sinaico-Tamaricetum jordanii Ighbareyeh, Cano-Ortiz \& Cano nova.

\begin{tabular}{|c|c|c|c|c|c|c|c|c|c|c|c|c|c|c|c|c|c|c|c|}
\hline Number of cluster & 104 & 108 & 110 & 124 & 128 & 130 & 143 & 147 & 149 & 163 & 191 & 195 & 197 & 211 & 215 & 217 & 230 & 234 & 236 \\
\hline Altitude in m & 620 & 573 & 630 & 611 & 578 & 580 & 433 & 416 & 428 & 496 & 466 & 563 & 412 & 459 & 475 & 510 & 475 & 490 & 470 \\
\hline Surface in $\mathbf{m}^{2}$ & 100 & 100 & 100 & 100 & 100 & 100 & 300 & 250 & 300 & 260 & 300 & 280 & 300 & 250 & 250 & 300 & 300 & 300 & 300 \\
\hline Cover rate \% & 75 & 75 & 75 & 75 & 75 & 60 & 60 & 75 & 75 & 75 & 75 & 75 & 75 & 75 & 75 & 75 & 80 & 65 & 60 \\
\hline Avarage height of veg. (m) & 3.5 & 3.3 & 2.5 & 2.5 & 2.5 & 2.5 & 5 & 5 & 5 & 4 & 4 & 4 & 4 & 4 & 4 & 5 & 4 & 4 & 3 \\
\hline Slope \% & 5 & 5 & 15 & 5 & 10 & 15 & 5 & 15 & 10 & 10 & 10 & 10 & 10 & 8 & 10 & 10 & 15 & 5 & 5 \\
\hline Orientation & W & W & S & $\mathrm{N}$ & $\mathrm{S}$ & $\mathrm{S}$ & $\mathrm{N}$ & W & $\mathrm{N}$ & W & $\mathrm{N}$ & $\mathrm{N}$ & $\mathrm{N}$ & $\mathrm{S}$ & $\mathrm{E}$ & $\mathrm{S}$ & W & W & $\mathrm{S}$ \\
\hline \multicolumn{20}{|l|}{ Characteristic of the association and upper units } \\
\hline Tamarix jordanis L. & 3 & 2 & 3 & 3 & 3 & 3 & 3 & 3 & 3 & 3 & 3 & 3 & 3 & 3 & 3 & 3 & 3 & 2 & 3 \\
\hline Capparis decidua (Forssk.) Edgew. & 2 & 2 & 2 & 2 & 2 & 2 & 2 & 2 & 2 & 2 & 2 & 2 & 2 & 2 & 2 & 2 & 2 & 2 & 2 \\
\hline Tamarix passerinoides Delile ex Desv. & 2 & 2 & 2 & 2 & 2 & 2 & 2 & 2 & 2 & 2 & 2 & 2 & 2 & 2 & 2 & 2 & 2 & 2 & 2 \\
\hline Salsola soda $\mathrm{L}$. & 3 & 3 & 3 & 3 & 3 & 3 & 3 & 3 & 3 & 3 & 3 & 3 & 3 & 3 & 3 & 3 & 3 & 3 & 3 \\
\hline Tamarix tetragyna Ehrenb. & 3 & 3 & . & 3 & 3 & 2 & 3 & 3 & . & 3 & 3 & 3 & . & 3 & 3 & 2 & 3 & 3 & . \\
\hline Tamarix parviflora DC. & 2 & 2 & . & 2 & 2 & 2 & 2 & 2 & . & 2 & 2 & 2 & . & 2 & 2 & 2 & 4 & 2 & . \\
\hline Tamarix palaestina Bertol & 2 & . & . & 2 & . & . & 2 & . & . & 2 & 2 & . & . & 2 & . & . & 4 & . & . \\
\hline Capparis sinaica Denne & 1 & . & . & 1 & . & . & 1 & . & . & 1 & 1 & . & . & 1 & . & . & 1 & . & . \\
\hline Crataegus sinaicus Boiss. & . & . & $\cdot$ & . & 2 & . & . & . & . & . & . & . & 3 & 3 & . & . & . & . & . \\
\hline Cercis siliquastrum L. & . & 2 & $\cdot$ & . & 2 & . & . & 2 & . & . & . & 2 & . & . & 2 & . & . & 2 & . \\
\hline
\end{tabular}

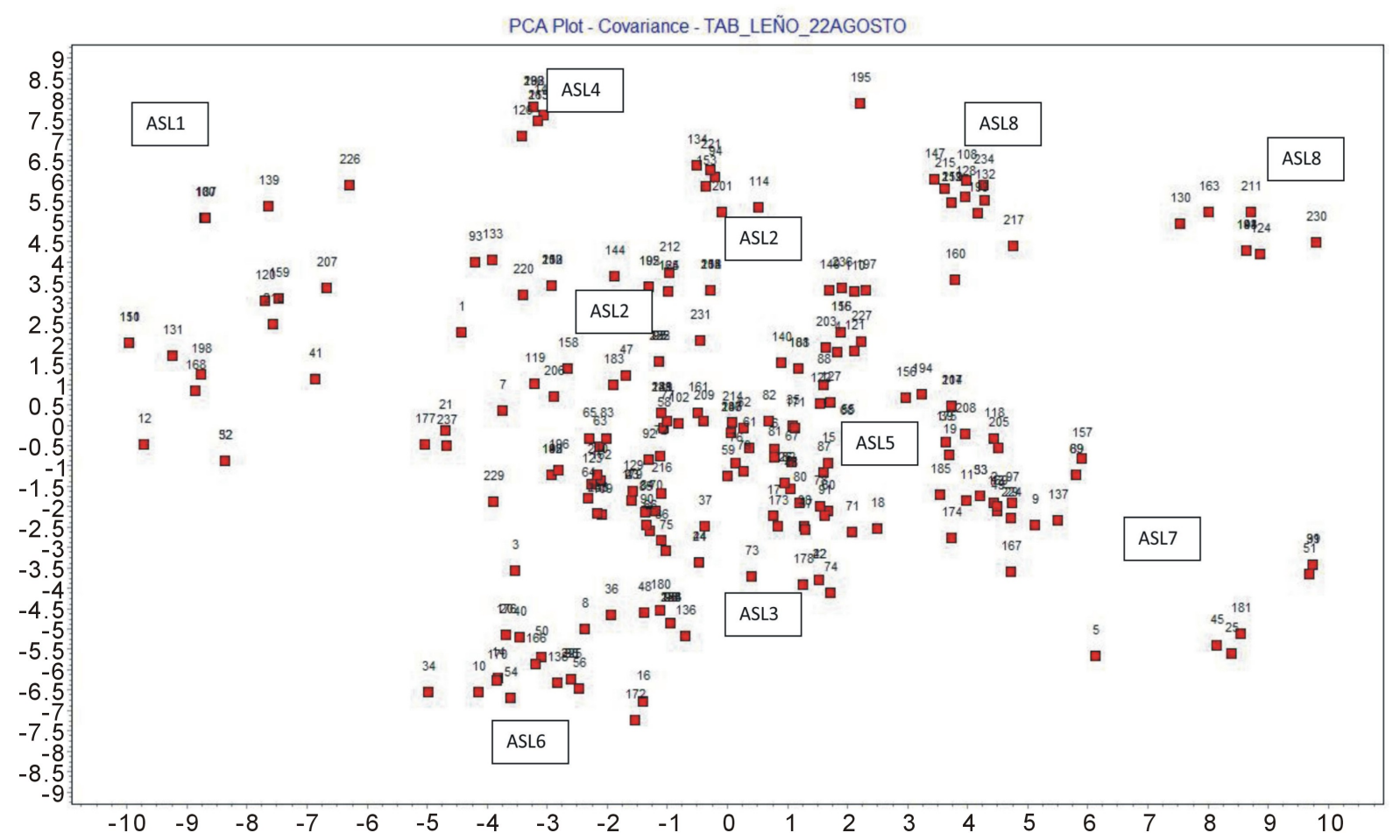

Figure 3. Principal component analyses.

Eurasia and Africa. Especially in the eastern southern corner of the Mediterranean Sea, creates unique geography and ecosystems which encountered endemic plants that do not exist in other places in the world and makes the introduced plants coexist strongly. Plants of three continents have interacted and spread throughout history. Consequently, this contribution to the rich diversity of Palestine flora has long captured the interest of ecologist and scientist alike.

Nevertheless, in Palestine, there are many challenges and abuses faced by the environment, biodiversity 
Table 10. Synthetic representation.

\begin{tabular}{|c|c|c|c|c|c|c|c|c|c|}
\hline Synthetic representation & ASL1 & ASL2 & ASL3 & ASL4 & ASL5 & ASL6 & ASL7 & ASL8 & \\
\hline Opuntia ficus-indica (L.) Mill & II & I & I & I & II & I & I & I & $\mathrm{N}$ \\
\hline Fagus crenata Blume & & & I & & II & I & II & I & $\mathrm{N}$ \\
\hline Capparis sinaica Denne & I & & I & & IV & & & II & $\mathrm{N}$ \\
\hline Abies cilicica (Antoine \& Kotschy) Carriere & I & II & I & $\mathrm{V}$ & I & I & III & & $\mathrm{N}$ \\
\hline Ceratonia siliqua L. & III & IV & I & $\mathrm{V}$ & I & III & I & IV & $\mathrm{N}$ \\
\hline Ficus carica L. Sp. & I & & II & II & I & IV & II & & $\mathrm{N}$ \\
\hline Rhamnus punctata Boiss. & II & & I & & I & I & & & $\mathrm{N}$ \\
\hline Galium incanum Sm. & I & IV & I & V & I & & & III & $\mathrm{N}$ \\
\hline Rhamnus lycioides Brot. & I & I & I & I & I & I & & & $\mathrm{N}$ \\
\hline Quercus calliprinos Webb. & & & I & & & I & I & I & $\mathrm{N}$ \\
\hline Cerasus prostrata Labill & I & I & III & I & & I & & II & $\mathrm{N}$ \\
\hline Cerasus microcarpa (C. A. Mey.) Boiss. & I & I & III & I & I & I & I & & $\mathrm{N}$ \\
\hline Quercus ithaburensi Kotschy. & & I & III & I & I & I & & I & $\mathrm{N}$ \\
\hline Rhus pentaphylla (Jacq.) Desf. & II & II & III & $\mathrm{V}$ & & & I & I & $\mathrm{N}$ \\
\hline Prunus dulcis (Mill.) D. A. Webb. & I & & I & & I & IV & II & & $\mathrm{N}$ \\
\hline Pteris vittata $\mathrm{L}$. & IV & $\mathrm{V}$ & II & II & I & & & & $\mathrm{N}$ \\
\hline Pistacia palaestina Boiss. & IV & $\mathrm{V}$ & I & & & I & I & & $\mathrm{E}$ \\
\hline Pistacia saportae Burnat & I & & I & & & & I & I & $\mathrm{N}$ \\
\hline Rhus tripartita Lobadium Raf. & I & & I & $\mathrm{V}$ & I & & & II & $\mathrm{N}$ \\
\hline Cercis siliquastrum L. & I & & I & & I & IV & I & I & $\mathrm{N}$ \\
\hline Pistacia atlantica Desf. & I & & I & I & I & & II & I & $\mathrm{N}$ \\
\hline Calligonum comosum $\mathrm{L}$. & I & & I & I & I & I & I & & $\mathrm{N}$ \\
\hline Rhus tripartita L. & I & & I & & I & III & & & $\mathrm{N}$ \\
\hline Arbutus andrachne L. & II & & I & & I & I & & & $\mathrm{E}$ \\
\hline Capparis decidua (Forssk.) Edgew. & III & I & I & & I & & & V & $\mathrm{E}$ \\
\hline Quercus look Kotschy & IV & & I & I & I & I & III & & $\mathrm{N}$ \\
\hline Quercus libani G. Olivier & I & & I & I & I & I & & & $\mathrm{N}$ \\
\hline Salix acmophylla Boiss & & & & III & I & I & & & $\mathrm{N}$ \\
\hline Pistacia lentiscus L. & I & & I & & I & I & I & & $\mathrm{N}$ \\
\hline Populus euphratica L. & II & I & I & II & I & I & & III & $\mathrm{N}$ \\
\hline Pinus halepensis Mill. & IV & & I & I & I & $\mathrm{V}$ & I & & $\mathrm{N}$ \\
\hline Periploca aphylla Tourn ex. L. & II & & I & & I & I & & I & $\mathrm{N}$ \\
\hline Physalis peruviana $\mathrm{L}$. & I & IV & I & I & I & & I & IV & $\mathrm{N}$ \\
\hline Cupressus sempervirens L. & I & I & I & I & I & I & V & III & $\mathrm{E}$ \\
\hline Morus nigra L. & I & & II & I & I & I & II & II & $\mathrm{N}$ \\
\hline Rubus fruticosus L. & & & I & I & I & I & II & I & $\mathrm{N}$ \\
\hline Rubus idaeus L. & I & & I & I & I & I & I & II & $\mathrm{N}$ \\
\hline Tamarix jordanis L. & I & I & I & I & I & I & II & IV & $\mathrm{E}$ \\
\hline Tamarix palaestina Bertol & & & II & & I & I & II & II & $\mathrm{E}$ \\
\hline Tamarix parviflora DC. & I & III & II & & I & & I & IV & $\mathrm{N}$ \\
\hline Tamarix passerinoides Delile ex Desv. & & II & I & & I & & V & V & $\mathrm{E}$ \\
\hline Tamarix tetragyna Ehrenb. & I & II & I & I & I & & V & IV & $\mathrm{E}$ \\
\hline Cytisopsis pseudocytisus (Boiss.) Fertig & & & II & & I & & V & & $\mathrm{E}$ \\
\hline Pyrus syriaca Boiss. & & & I & I & I & & & I & $\mathrm{E}$ \\
\hline Rhus coriaria $\mathrm{L}$. & I & I & I & I & I & I & & I & $\mathrm{N}$ \\
\hline Celtis australis L. & I & I & I & & & I & I & I & $\mathrm{N}$ \\
\hline Sorbus torminalis (L.) Crantz & I & I & I & & I & I & I & & $\mathrm{N}$ \\
\hline
\end{tabular}




\section{Continued}

\begin{tabular}{|c|c|c|c|c|c|c|c|c|c|}
\hline Crataegus sinaicus Boiss. & I & & I & & I & & & I & $\mathrm{E}$ \\
\hline Solanum incanum L. & & I & I & I & I & I & I & I & $\mathrm{E}$ \\
\hline Solanum villosum Miller. & & & I & & $\mathrm{I}$ & & $\mathrm{I}$ & & $\mathrm{N}$ \\
\hline Calotropis procera (Aiton) W. T. Aiton & $\mathrm{I}$ & IV & I & & $\mathrm{I}$ & & & & $\mathrm{E}$ \\
\hline Salsola orientalis S. G. Gmel & II & II & I & & I & & II & & $\mathrm{E}$ \\
\hline Salsola soda L. & II & & I & & $\mathrm{I}$ & & & I & $\mathrm{N}$ \\
\hline Salsola stocksii Boiss. & & & I & & I & & III & & $\mathrm{E}$ \\
\hline Dalbergia sissoo Roxb. & I & & I & II & $\mathrm{I}$ & & & & $\mathrm{N}$ \\
\hline Bryonia dioica Jacq. & III & I & I & I & I & I & & & $\mathrm{N}$ \\
\hline Bryonia syriaca Boiss. & I & & I & & & I & & I & $\mathrm{N}$ \\
\hline Phoenix dactylifera L. & & & & & & I & & & $\mathrm{N}$ \\
\hline
\end{tabular}

Percentage of plant species presence in the samples and communities: $\mathrm{V}=100 \%$, IV $=60.1 \%-80 \%, \mathrm{III}=40.1 \%-60 \%$, II $=20.1 \%-40 \%$ and $\mathrm{I}=0.1 \%-$ 20\%. N: Native and E: Endemic. Association (ASL), ASL1-Pistacio palaestinae-Quercetum lokii. ASL2-Capparido sinaicae-Ceratonietum siliquae. ASL3-Cerasus microcarpae-Quercetum ithaburensis. ASL4_Pyro siriacae-Abietetum cilicicae. ASL5-Abio ciliciae-Ceratonietum siliquae. ASL6-Periploco aphylli-Pinetum halepensis. ASL7-Cytisopsis pseudocytiso-Tamaricetum tetragynae. ASL8-Crataego sinaicae-Tamaricetum jordanii.

and diversity, the economy and human life and many factors of result of the conflict with the Israeli occupation. Certainly the situation is far from optimistic. However, work is already underway to reverse the negative trend of the last 20 - 30 years.

As a result of the phytosociological research conducted in the village of Idna, located in the region of Hebron, we describe eight new plant associations, of which two have a dry-sub-humid ombrotype (Pistacio palaestinaeQuercetum lokii; Cerasus microcarpae-Quercetum ithaburensis). The places where both associations are located are optimal for the cultivation of olive. We propose that both plant communities should be considered as indicators for olive cultivation. The area occupied by the meso-Mediterranean and humid association Pyro siriacae-Abietetum cilicicae is also suitable for olive cultivation, but using more disease-resistant varieties. Conversely, associations located in arid, semi-arid and lower dry environments cannot be considered useful for olive cultivation, although less dry areas may be used for growing grapevines. We propose a change of crop for the arid and semi-arid areas; we propose the cultivation of Argania spinosa (Sapotaceae), and of aromatic species with a commercial value.

\section{References}

[1] Ighbareyeh, J.M.H., Cano-Ortiz, A. and Cano, E. (2014) Biological and Bioclimatic Basis to Optimize Plant Production: Increased Economic Areas of Palestine. Agricultural Science Research Journal, 4, 10-20.

[2] Ighbareyeh, J.M.H., Cano-Ortiz, A. and Cano, E. (2014) Case Study: Analysis of the Physical Factors of Palestinian Bioclimate. American Journal of Climate Change, 3, 223-231. http://dx.doi.org/10.4236/ajcc.2014.32021

[3] Zohary, M. (1962) Plant Life of Palestine. Ronald Press Company, New York.

[4] Shimida, A. (1995) General References on Biodiversity and Theory of Ecological Richness, Especially Concerned with Arid and Mediterranean Ecosystems. The Hebrew University, Jerusalem.

[5] Médail, F. and Quézel, P. (1997) Hot-Spots Analysis for conservation of Plant Biodiversity in the Mediterranean Basin. Annals of the Missouri Botanical Garden, 84, 112-127. http://dx.doi.org/10.2307/2399957

[6] Myers, N. and Cowling, R. (1999) Mediterranean Basin. In: Mittermeier, R.A., Myers, N. and Goettsch Mittermeier, C. Eds., Hotspots - Earth's Biologically Richest and Most Endangered Terrestrial Ecoregions, CEMEX \& Conservation International, Mexico City, 254-267.

[7] Danin, A. (2004) Distribution Altas of Plants in Flora Palaestina Area. 2nd Edition, Academy of science and Humanities, Jerusalem.

[8] Sawalha, Kh., Ed. (2005) Plant Biodiversity. Al-Quds University, Jerusalem.

[9] Braun-Blanquet, J. (1979) Fitosociología. Bases para el estudio de las comunidades vegetales. Blume, Madrid, 820 p.

[10] Van Der Maarel, E. (1979) Transformation of Cover-Abundance Values in Phytosociology and Its Effects on Community Similarity. Vegetatio, 39, 97-114. http://dx.doi.org/10.1007/BF00052021

[11] Shaltout, K.H., Sheded, M.G., El-Kady, H.F. and Al-Sodany, Y.M. (2003) Phytosociology and Size Structure of Nitra- 
ria retusa along the Egyption Red Sea Coast. Journal of Arid Environments, 53, 331-345. http://dx.doi.org/10.1006/jare.2002.1054

[12] Alhamad, M.N. (2006) Ecological and Species Diversity of Arid Mediterranean Grazing Land Vegetation. Journal of Arid Environments, 66, 698-715. http://dx.doi.org/10.1016/j.jaridenv.2006.01.001

[13] Djamali, M., Brewer, S., Breckler, S.W. and Jackson, S.T. (2012) Climate Determinism in Phytogeographic Regionalization: A Test from the Irano-Turanian Region, SW and Central Asia. Flora-Morphology, Distribution, Functional Ecology of Plants, 207, 237-249. http://dx.doi.org/10.1016/j.flora.2012.01.009

[14] Biondi, E. (2011) Phytosociology Today: Methodological and Conceptual Evolution. Plant Biosystems, 145, 19-29. http://dx.doi.org/10.1080/11263504.2011.602748

[15] Pott, R. (2011) Phytosociology: A Modern Geobotanical Method. Plant Biosystems, 145, 9-18. http://dx.doi.org/10.1080/11263504.2011.602740

[16] Rivas-Martínez, S. (2004) Sinopsis biogeográfica, bioclimática y vegetacional de América del Norte. Fitosociología, 41, 19-52.

[17] Rivas-Martínez, S. (2005) Notions on Dynamic-Catenal Phytosociology as a Basis of Landscape Science. Plant Biosystems, 139, 135-144. http://dx.doi.org/10.1080/11263500500193790

[18] Applied Research Institute-Jerusalem (ARIJ) (2006-2009) GIS Database. http://vprofile.arij.org/hebron/pdfs/Al\%20Heila pro.pdf

[19] Taher, N.A.B.A. and Von Jaffa, K.S. (2009) Loggerhead Sea Turtle (Caretta caretta Linnaeus, 1758) Nesting in Palestine. Gazelle: The Palestinian Biological Bulletin, 1-31.

[20] Braun-Blanquet, J. and Bolos, O. (1958) Les groupements végétaux du bassin moyen de l'Ebre et leur dynamisme. Anales Aula Dei, 5, 1-266.

[21] Braun-Blanquet, J. (1952) Les groupements vegetaux del France meditérranéenne. Editions C.N.R.S., Montpellier.

[22] Braun-Blanquet, J. (1952) Irradiations europeennes dans la vegetation de la Kroumirie. Vegetatio, 4, 182-194. http://dx.doi.org/10.1007/BF00297018

[23] Rivas-Martínez, S., Díaz, T.E., Fernández-González, F., Izco, J., Loidi, J., Lousa, M. and Penas, E. (2002) Vascular Plant Communities of Spain and Portugal. Itinera Geobotanica, 15, 433-922.

[24] Rivas-Martínez, S., Biondi, E., Costa, M. and Mossa, L. (2003) Datos sobre la vegetación de la clase Quercetea ilicis en Cerdeña. Fitosociologia, 40, 35-38.

[25] Rivas-Martínez, S., Penas, A. and Dìaz, T.E. (2001) Biogeographic Map of Europe (Scale 1:16,000,000). Cartographic Service, University of León, León.

[26] Zohary, M. (1966) Flora Palaestina. Part 1, Text Equisetaceae to Moringaceae. Israel Academy of Science and Humanities, Jerusalem, 346.

[27] Zohary, M. (1972) Flora Palaestina. Part 2, Text Platanaceae to Umbelliferae. Israel Academy of Science and Humanities, Jerusalem, 656.

[28] Dothan, F.N. (1978) Flora Palaestina, Part Three, Text Ericaceae to Compositae. Israel Academy of Science and Humanities, Jerusalem, 481.

[29] Dothan, F.N. (1986) Flora Palaestina, Part Four Plates, Text Alismtaceae to Orchidaceae. Academy of Science and Humanities, Jerusalem, 525.

[30] Danin, A. and Orshan, G. (1999) Vegetation of Israel: I. Desert and Coastal Vegetation. Buckhuys, Leiden, 341.

[31] Danin, A. and Feinbrun-Dothan, N. (1991) Analytical Flora of Eretz-Israel. CANA Publishing House Ltd., Jerusalem.

[32] Rivas-Martínez, S. (1975) La vegetación de la clase Quercetea ilicis en España y Portugal. Anales Instituto Botánico Cavanilles, 31, 205-262.

[33] Molinier, R. (1934) Études phytosociologiques et écologiques en Provence occidentale. Anales Museo Historia Natural Marseille, 27, 1-273.

[34] Molinier, R. (1968) Le dynamisme de la végétation provençale. Collectanea Botánica, 7, 817-844.

[35] Molinier, R. (1973) Les études phytosociologiques en Provencem cristalline. Boletín Museo Historia Natural Marseille, 33, 1-46.

[36] Danin, A. (2000) The Nomenclature News of flora Palaestina. Flora Mediterranean, 10, 109-127. 
Associations described as new:

Pistacio palaestinae-Quercetum lokii*

Capparido sinaicae-Ceratonietum siliquae

Cerasus microcarpae-Quercetum ithaburensis*

Pyro siriacae-Abietetum cilicicae*

Abio ciliciae-Ceratonietum siliquae

Periploco aphylli-Pinetum halepensis

Cytisopsis pseudocytiso-Tamaricetum tetragynae

Crataego sinaicae-Tamaricetum jordanii

*Associations in which olive cultivation is possible. 
Scientific Research Publishing (SCIRP) is one of the largest Open Access journal publishers. It is currently publishing more than 200 open access, online, peer-reviewed journals covering a wide range of academic disciplines. SCIRP serves the worldwide academic communities and contributes to the progress and application of science with its publication.

Other selected journals from SCIRP are listed as below. Submit your manuscript to us via either submit@scirp.org or Online Submission Portal.
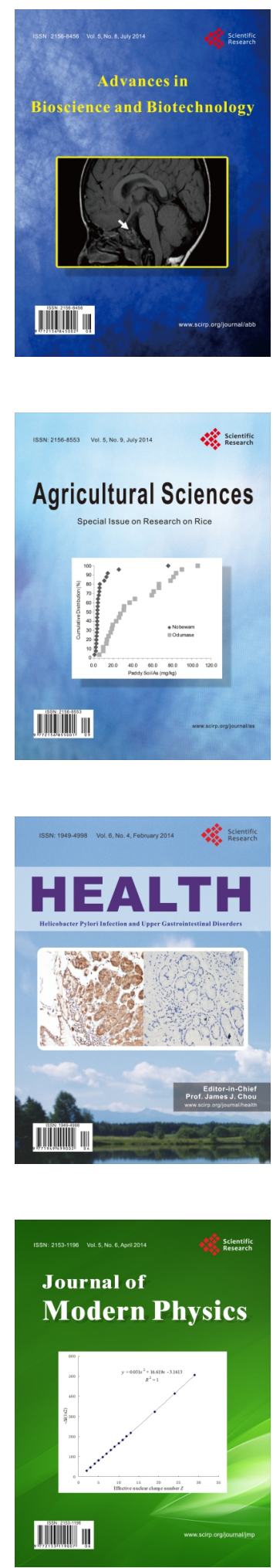
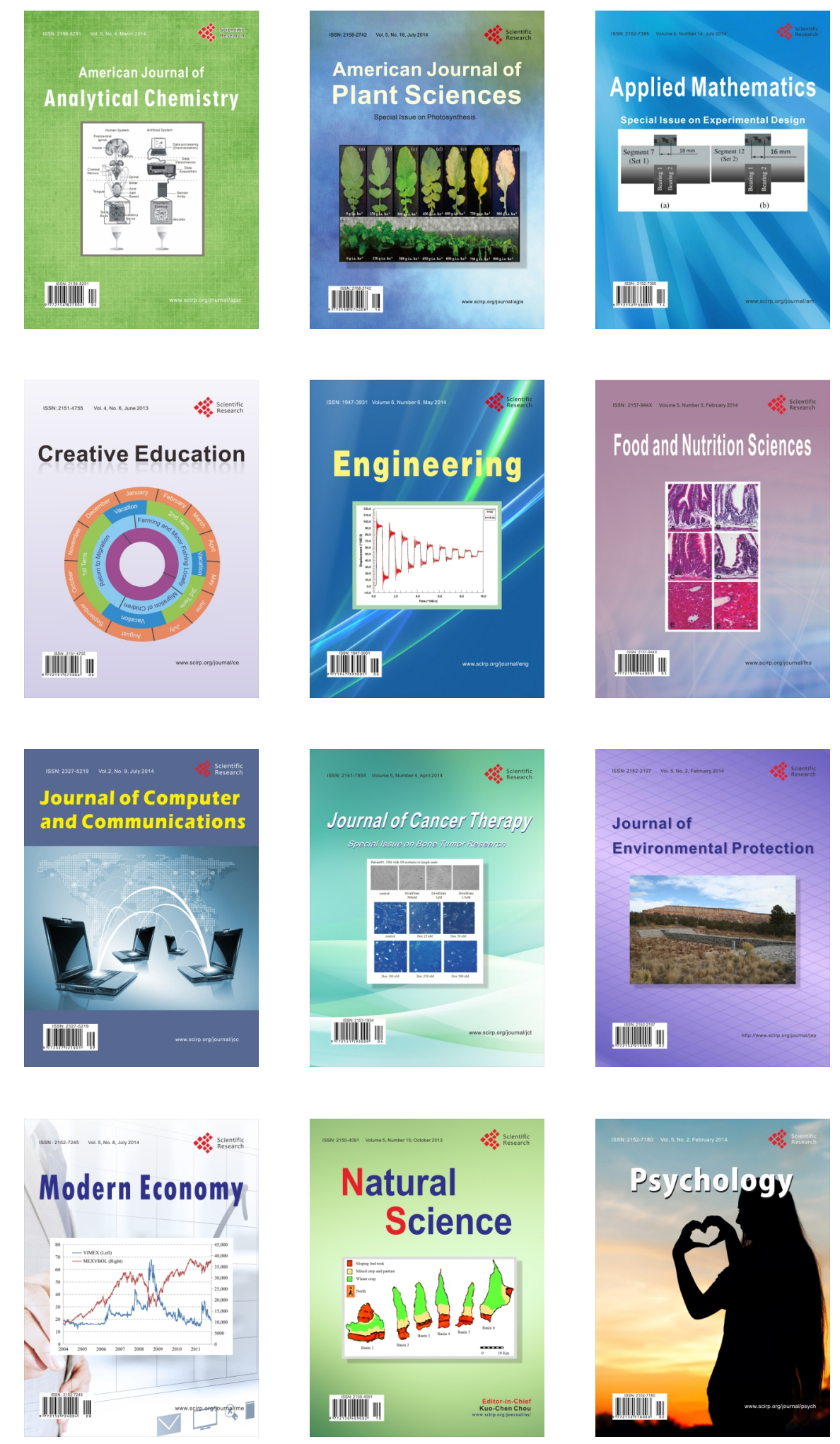